International Journal of Pure and Applied Mathematics

Volume 82 No. 5 2013, 683-693

ISSN: 1311-8080 (printed version); ISSN: 1314-3395 (on-line version)

url: http://www.ijpam.eu

doi: http://dx.doi.org/10.12732/ijpam.v82i5.2

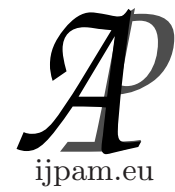

\title{
A STOCHASTIC MODEL FOR ESTIMATION OF EXPECTED \\ TIME TO RECRUITMENT WITH TWO SOURCES OF DEPLETION OF MAN POWER UNDER CORRELATED INTERARRIVAL TIMES
}

\author{
K. Usha ${ }^{1}$, P. Leelathilagam ${ }^{2}$, R. Ramanarayanan ${ }^{3}$ \\ ${ }^{1}$ Department of Mathematics \\ Queen Marry's College \\ Chennai, 600004, INDIA \\ ${ }^{2}$ Department of Mathematics \\ Vivekanandha College of Engineering for Women \\ Thiruchengode, 637205, INDIA \\ ${ }^{3}$ VelTech., Dr. Rangarajan., Dr. Sakunthala. \\ Technical University \\ Chennai, 600062, INDIA
}

\begin{abstract}
Leaving of personnel in any organization is a common phenomenon. This results in the loss of manpower and recruitment is suggested to make up the manpower deficiency. At the same time it may be observed that frequent recruitments are not suggested since it involves cost and time. Hence the process of recruitment is postponed till the cumulative loss of manpower crosses a particular level known as threshold. Determination of the expected time to recruitment is an important aspect of studying manpower planning. In this paper the expression for expected time to recruitment is obtained under the assumptions that the interarrival times between the successive epochs of decision making are constantly correlated where as interarrival times between the successive epochs of transfer of personnel to sister organizations are i.i.d randomvariables.
\end{abstract}

Received: July 15, 2012

(C) 2013 Academic Publications, Ltd. url: www.acadpubl.eu

$\S_{\text {Correspondence author }}$ 
AMS Subject Classification: $62 \mathrm{NO} 5$

Key Words: recruitment, depletion of man power, policy decisions, transfer of personnel, threshold

\section{Introduction}

In an organization, the depletion of manpower takes place due to two different cases:

1) whenever the policy decisions regarding pay, perquisites and work schedule are revised and

2) due to transfer of personnel to the other organization of the same management. When the shortage of manpower occurs, immediate recruitment cannot be carried out due to the expenditure involved. Hence the depletion of manpower is allowed till the total magnitude of depletion crosses a particular level called the threshold. As and when the total depletion of manpower crosses the threshold level the process of recruitment is started . The Authors [5] have discussed a stochastic model for estimating the expected time to recruitment under the assumptions that i) the depletion of manpower is in terms of persons leaving at every epoch of decision making and at every epoch of transfer and hence they are in terms of discrete random variables. ii) the recruitment is carried out as and when the total depletion crosses a level called the threshold which is a discrete random variable.

In this paper it is proposed to determine the expected time to recruitment time under the assumption that the interarrival times between successive epochs of policy decisions are not independent but correlated, whereas the interarrival times between successive epochs of transfers are i.i.d random variables.

In obtaining the expression for expected time to recruitment the result due to Gurland [2] has been used .Shock model approach to determine the expected time for Recruitment has been discussed by R. Sathiyamoorthy and R.Elangovan [3]. R.Sathiyamoorthy and S.Pathasarathy [4] have obtained the expression for the expected time to Recruitment in a two grade organization using shock model approach.

\section{Assumptions}

1. In an organization, The depletion of manpower occurs whenever the policy decisions are announced. The interarrival times between decision epochs are 
random variables having exponential distribution with parameter a . These random variables are constantly correlated and exchangeable but not independent.

2. The depletion of man power also occurs due to transfer of personnel and the interarrival times between successive epochs of transfer are i.i.d random variables having exponential distribution with parameter $\lambda$.

3. As and when the total depletion due to the above two reasons crosses the threshold which itself is a random variable, recruitment becomes necessary.

4. The two types of depletion are linear and additive.

\section{Notations}

$X_{i^{-}}$a continuous random variable representing the amount of depletion of man hours due to the $i^{t h}$ epoch of policy decisions. $X_{i}^{\prime} s$ are i.i.d random variables having exponential distribution with parameter $\alpha$.

$Y_{j}$ - a continuous random variable representing the amount of depletion of man hours due to the $j^{\text {th }}$ event of transfer of personnel. $Y_{j}^{\prime} s$ are i.i.d random variables having exponential distribution with parameter $\mu$.

$$
\begin{aligned}
& h(.)=\text { p.d.f of } X_{i} \\
& k(.)=\text { p.d.f of } Y_{j}
\end{aligned}
$$

$u_{i}$ - a random variable denoting the interarrival times between decision epochs. $u_{i}^{\prime} s$ are constantly correlated and exchangeable random variables with exponential distribution with parameter $a$.

$v_{j}$ - a random variable representing the interarrival times between the successive epochs of transfer . $v_{j}^{\prime} s$ are i.i.d random variables with exponential distribution with parameter $\lambda$.

$$
\begin{aligned}
& f(.)=\text { p.d.f of } u_{i} \\
& g(.)=\text { p.d.f of } v_{j}
\end{aligned}
$$

$\mathrm{Z}$ - a continuous r.v representing the threshold level and is assumed to be exponential with parameter $\mathrm{c}$.

$F_{m}()=$. c.d.f of $\sum_{i=0}^{m} u_{i}$, the sum of the interarrival times between decision making epochs. 
of transfers.

$G_{n}()=$. c.d.f of $\sum_{j=1}^{n} v_{j}$, the sum of the interarrival times between the epochs

$T$ - a r.v denoting the time to recruitment in the organizations.

$\tilde{X}=X_{1}+X_{2}+\ldots+X_{m} \quad \tilde{Y}=Y_{1}+Y_{2}+\ldots+Y_{n}$

$L(t)=P(T<t)=$ c.d.f of the time to recruitment of the system.

$S(t)=$ Survivor function $P(T>t)$

$L^{*}(S)=$ Laplace transform of $L(t)$

$h^{*}()=$. Laplace transform of $h($.

$k^{*}()=$. Laplace transform of $k($.

The probability that the total depletion of manpower on " $m$ " occasions of decision making and " $n$ " occasions of transfer of personnel does not exceed the threshold level is given as

$$
\begin{aligned}
P[\tilde{X}+\tilde{Y}<Z] & =\int_{0}^{\infty} Q_{\tilde{x}+\tilde{y}}(z) c . e^{-c z} d z \\
& =c \int_{0}^{\infty} Q_{\tilde{x}+\tilde{y}}(z) e^{-c z} d z \\
& =c Q_{\tilde{x}+\tilde{y}}^{*}(c)
\end{aligned}
$$

But we know that

$$
F_{m}^{*}(s)=\frac{1}{s} f_{(m)}^{*}(s)
$$

Using this result

$$
\begin{aligned}
P[\tilde{X}+\tilde{Y}<Z] & =c \frac{q_{\tilde{x}+\tilde{y}}^{*}(c)}{c} \\
& =q_{\tilde{x}}^{*}(c) q_{\tilde{y}}^{*}(c) \\
& =\left[q^{*}(c)\right]^{m}\left[q^{*}(c)\right]^{n} \\
& =\left(h^{*}(c)\right)^{m}\left(k^{*}(c)\right)^{n}
\end{aligned}
$$

Since $\mathrm{X}$ and $\mathrm{Y}$ are independent and $X_{i}$ and $Y_{j}$ are i.i.d random variable.

\section{Main Results}

$S(t)=P(T>t)=$ Prob that there are exactly " $\mathrm{m}$ " occasions of policy making and " $n$ " occasions of transfer and the total depletion does not cross the threshold 
$Z$ in $(0, t)$.

$$
\begin{aligned}
= & \sum_{m=0}^{\infty}\left(F_{m}(t)-F_{m+1}(t)\right)\left(h^{*}(c)\right)^{m} \sum_{n=0}^{\infty}\left(G_{n}(t)-G_{n+1}(t)\right)\left(k^{*}(c)\right)^{n} \\
S(t)= & \left\{1-\left(1-h^{*}(c)\right)\left[\sum_{m=1}^{\infty} F_{m}(t)\left(h^{*}(c)\right)^{m-1}\right]\right\} \\
& \left\{1-\left(1-k^{*}(c)\right)\left[\sum_{n=1}^{\infty} G_{n}(t)\left(k^{*}(c)\right)^{n-1}\right]\right\}
\end{aligned}
$$

Now

$$
\begin{aligned}
L(t)= & P(T<t)=1-S(t) \\
= & \left(1-h^{*}(c)\right)\left[\sum_{m=1}^{\infty} F_{m}(t)\left(h^{*}(c)\right)^{m-1}\right] \\
+ & \left(1-k^{*}(c)\right)\left[\sum_{n=1}^{\infty} G_{n}(t)\left(k^{*}(c)\right)^{n-1}\right] \\
& -\left(1-h^{*}(c)\right)\left(1-k^{*}(c)\right) \\
& {\left[\sum_{m=1}^{\infty} F_{m}(t)\left(h^{*}(c)\right)^{m-1} \sum_{n=1}^{\infty} G_{n}(t)\left(k^{*}(c)\right)^{n-1}\right] }
\end{aligned}
$$

Pdf is given by

$$
\begin{aligned}
l(t)= & \left(1-h^{*}(c)\right) \sum_{m=1}^{\infty} f_{m}(t)\left(h^{*}(c)\right)^{m-1}+\left(1-k^{*}(c)\right) \sum_{n=1}^{\infty} g_{n}(t)\left(k^{*}(c)\right)^{n-1} \\
& -\left(1-h^{*}(c)\right)\left(1-k^{*}(c)\right)\left[\sum_{m=1}^{\infty} f_{m}(t)\left(h^{*}(c)\right)^{m-1} \sum_{n=1}^{\infty} G_{n}(t)\left(k^{*}(c)\right)^{n-1}\right. \\
& \left.+\sum_{m=1}^{\infty} F_{m}(t)\left(h^{*}(c)\right)^{m-1} \sum_{n=1}^{\infty} g_{n}(t)\left(k^{*}(c)\right)^{n-1}\right] \\
= & \left(1-h^{*}(c)\right) \sum_{m=1}^{\infty} f_{m}(t)\left(h^{*}(c)\right)^{m-1} \\
& +\left(1-k^{*}(c)\right) \sum_{n=1}^{\infty} \lambda e^{-\lambda t} \frac{(\lambda t)^{n-1}}{(n-1) !}\left(k^{*}(c)\right)^{n-1} \\
& -\left(1-h^{*}(c)\right)\left(1-k^{*}(c)\right)\left[\sum_{m=1}^{\infty} f_{m}(t)\left(h^{*}(c)\right)^{m-1}\right.
\end{aligned}
$$




$$
\begin{aligned}
& \sum_{n=1}^{\infty} \int_{0}^{t} \frac{\lambda e^{-\lambda t}(\lambda t)^{n-1}}{(n-1) !}\left(k^{*}(c)\right)^{n-1} d t \\
& \left.+\sum_{m=1}^{\infty} F_{m}(t)\left(h^{*}(c)\right)^{m-1} \sum_{n=1}^{\infty} \lambda e^{-\lambda t} \frac{(\lambda t)^{n-1}}{(n-1) !}\left(k^{*}(c)\right)^{n-1}\right] \\
& =\left(1-h^{*}(c)\right) \sum_{m=1}^{\infty} f_{m}(t)\left(h^{*}(c)\right)^{m-1} \\
& +\left(1-k^{*}(c)\right) \lambda e^{-\lambda t\left(1-k^{*}(c)\right)} \\
& -\left(1-h^{*}(c)\right)\left(1-k^{*}(c)\right) \sum_{m=1}^{\infty} f_{m}(t)\left(h^{*}(c)\right)^{m-1}\left[\frac{1-e^{-\lambda t\left(1-k^{*}(c)\right)}}{1-\left(k^{*}(c)\right)}\right] \\
& -\sum_{m=1}^{\infty} F_{m}(t)\left(h^{*}(c)\right)^{m-1} \lambda e^{-\lambda t\left(1-k^{*}(c)\right)}\left(1-h^{*}(c)\right)\left(1-k^{*}(c)\right) \\
& =\left(1-k^{*}(c)\right) \lambda e^{-\lambda t\left(1-k^{*}(c)\right)} \\
& +\left(1-h^{*}(c)\right) \sum_{m=1}^{\infty} f_{m}(t)\left(h^{*}(c)\right)^{m-1} e^{-\lambda t\left(1-k^{*}(c)\right)} \\
& -\sum_{m=1}^{\infty} F_{m}(t)\left(h^{*}(c)\right)^{m-1} \lambda e^{-\lambda t\left(1-k^{*}(c)\right)}\left(1-h^{*}(c)\right)\left(1-k^{*}(c)\right) \\
& l^{*}(s)=\frac{\lambda\left(1-k^{*}(c)\right)}{s+\lambda\left(1-k^{*}(c)\right)} \\
& +\left(1-h^{*}(c)\right) \sum_{m=1}^{\infty} f_{m}^{*}\left(s+\lambda\left(1-k^{*}(c)\right)\left(h^{*}(c)\right)^{m-1}\right. \\
& -\frac{\lambda\left(1-h^{*}(c)\right)\left(1-k^{*}(c)\right)}{s+\lambda\left(1-k^{*}(c)\right)} \sum_{m=1}^{\infty} f_{m}^{*}\left(s+\lambda\left(1-k^{*}(c)\right)\right)\left(h^{*}(c)\right)^{m-1} \\
& -\left.\frac{d l^{*}(s)}{d s}\right|_{s=0}=\frac{1}{\lambda\left(1-k^{*}(c)\right)}-\sum_{m=1}^{\infty} f_{m}^{*}\left(\lambda\left(1-k^{*}(c)\right)\right) \\
& \left(h^{*}(c)\right)^{m-1}\left[\frac{\lambda\left(1-h^{*}(c)\right)\left(1-k^{*}(c)\right)}{\lambda^{2}\left(1-k^{*}(c)\right)^{2}}\right] \\
& E(T)=\frac{1}{\lambda\left(1-k^{*}(c)\right)}-\frac{\left(1-h^{*}(c)\right)}{\lambda\left(1-k^{*}(c)\right)} \sum_{m=1}^{\infty} f_{m}^{*}\left(\lambda\left(1-k^{*}(c)\right)\left(h^{*}(c)\right)^{m-1}\right.
\end{aligned}
$$


Gurland [2] has obtained the expression for the c.d.f of the partial sum $S_{m}=$ $u_{1}+u_{2}++u_{m}$.

When the Random variables $u_{i} \quad i=1,2, m$ are exchangeable, exponentially distributed and are with constant correlation R.Gurland has derived that the c.d.f of

$S_{m}=U_{1}+U_{2}++U_{m}$ is $F_{m}(x)=P\left(S_{m} \leq x\right)$

$$
=(1-R) \sum_{i=0}^{\infty} \frac{(m R)^{i}}{(I-R+m R)^{i+1}} \frac{\psi\left(m+i, \frac{x}{b}\right)}{(m+i-1) !}
$$

where $\psi(n, x)=\int_{0}^{x} e^{-x} x^{n-1} d x$ and $b=a(1-R)$ and $f_{m}^{*}(S)=$ Laplace tranform of $f_{m}(x)$ is

$$
f_{m}^{*}(s)=\frac{1}{(1+b s)^{m}\left(1+\frac{m R b s}{(1-R)(1+b s)}\right)}
$$

where $b=a(1-R)$, a being the parameter of the exponential distribution.

Noting that the interarrival times between decision making epochs are constantly correlated exponential variables with parameter a .

$$
f_{m}^{*}\left(\lambda\left(1-k^{*}(c)\right)\right)=\frac{(1-R)\left[1+b \lambda\left(1-k^{*}(c)\right)\right]^{1-m}}{\left(1-R+\left(1-k^{*}(c)\right)[b \lambda-b \lambda R+m R b \lambda]\right.}
$$

$h($.$) and k($.$) are exponentially distributed with parameters \alpha, \mu$

$$
\begin{aligned}
& h^{*}(c)=\frac{\alpha}{\alpha+c} \\
& k^{*}(c)=\frac{\mu}{\mu+c} \\
& 1-h^{*}(c)=\frac{c}{c+\alpha} \\
& 1-k^{*}(c)=\frac{c}{c+\mu} \\
& E(T)=\frac{1}{\lambda\left(\frac{c}{c+\mu}\right)}-\frac{\frac{c}{c+\alpha}}{\lambda\left(\frac{c}{c+\mu}\right)} \sum_{m=1}^{\infty} \frac{(1-R)\left[1+b \lambda\left(1-k^{*}(c)\right)\right]^{1-m}\left(h^{*}(c)\right)^{m-1}}{\left(1-R+\left(\frac{c}{c+\mu}\right)[b \lambda-b \lambda R+m R b \lambda]\right.} \\
& =\frac{c+\mu}{c \lambda}-\frac{(c+\mu)^{2}(1-R)}{\lambda(c+\alpha)} \sum_{m=1}^{\infty} \frac{\left(\frac{c+\mu}{c+\mu+b \lambda c}\right)^{m-1}\left[\frac{\alpha}{\alpha+c}\right]^{m-1}}{(c+\mu)(1-R)+b \lambda c(1-R+m R)}
\end{aligned}
$$


From this we can find expectation of time to recruitment. As the serious is infinite, it is tedious to find out. We can also have the finite occasions of decision making and transfer of personnels as a special case.

Suppose two occasions of policy decisions and $\mathrm{n}$ occasions of transfer of personnel are considered.

Put $\mathrm{m}=2$

$$
\begin{aligned}
& E(T)=\frac{c+\mu}{c \lambda}-\frac{(c+\mu)^{2}(1-R)}{\lambda(c+\alpha)} \\
& {\left[\frac{1}{(1-R)(c+\mu)+b \lambda c}+\frac{(c+\mu) \alpha}{(c+\mu+b \lambda c)(\alpha+c)((c+\mu)(1-R)+b \lambda c(1+R))}\right]}
\end{aligned}
$$

We discuss some numerical examples for the above case.

\section{Numerical Example}

$\lambda=1.2 \quad \alpha=1 \quad b=0.24 \quad C=0.24 \quad \mu=2$

Table : 1

\begin{tabular}{|c|c|}
\hline$R$ & $E(T)$ \\
\hline-0.2 & 5.1561 \\
\hline 0 & 5.175 \\
\hline 0.2 & 5.2027 \\
\hline 0.4 & 5.2474 \\
\hline 0.6 & 5.3319 \\
\hline
\end{tabular}

\section{Conclusion}

As a value of $\alpha$ which is the parameter of the random variable representing the amount of depletion at every epoch of policy decision increases then it is seen that $E(T)$ which is the expected time to recruitment increases. This is due to the fact that $X$ is distributed as exponential with parameter $\alpha$ and $E(X)=\frac{1}{\alpha}$

As $\alpha$ increases $E(X)$ namely the average amount of depletion decreases hence $E(T)$ namely time to recruitment increases. 
Graph : 1

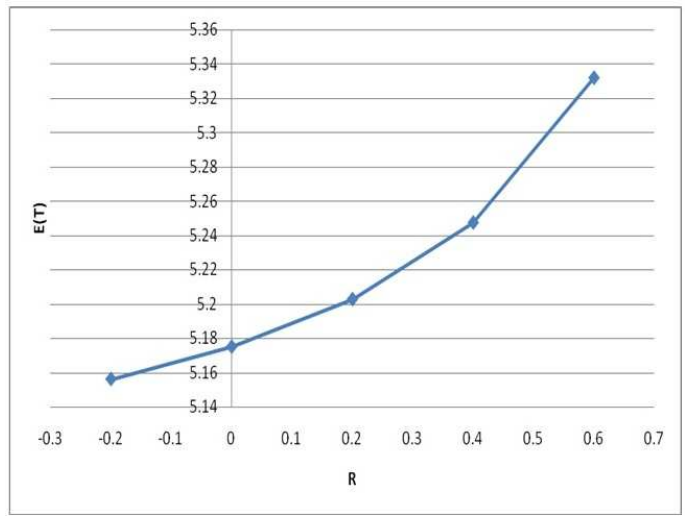

Table : 2

$\lambda=1.2 \quad \mathrm{R}=0.8 \quad \mathrm{~b}=0.24 \quad \mathrm{C}=0.24$ $\alpha=1$

\begin{tabular}{|c|c|}
\hline$\mu$ & $\mathrm{E}(\mathrm{T})$ \\
\hline 1 & 3.2298 \\
\hline 2 & 6.5459 \\
\hline 3 & 7.8403 \\
\hline 4 & 10.1158 \\
\hline 5 & 16.8199 \\
\hline
\end{tabular}

\section{Graph : 2}

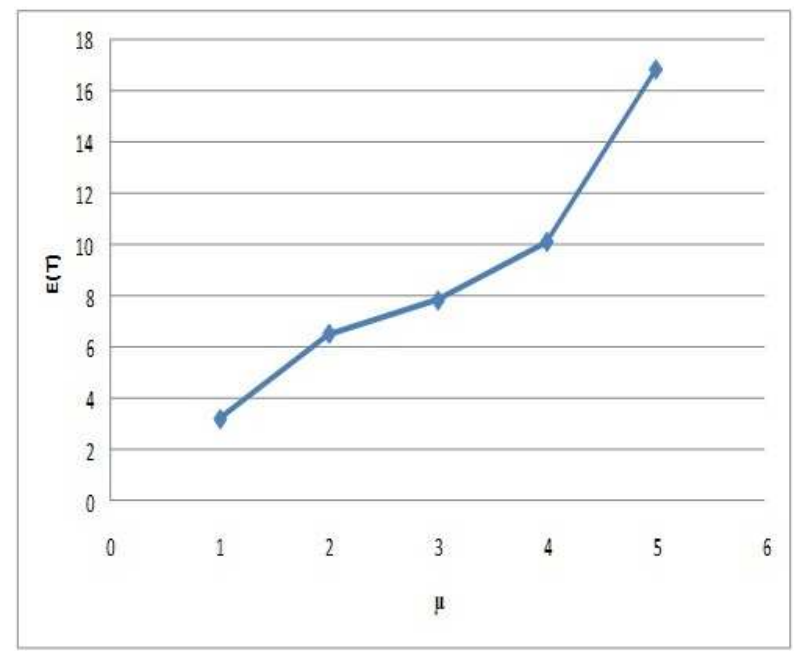


Table: 3

$\lambda=1.2 \quad \mathrm{~b}=0.24 \quad \mathrm{C}=0.24$

$\mu=1 \quad \mathrm{R}=0.8$

\begin{tabular}{|c|c|}
\hline$\alpha$ & $\mathrm{E}(\mathrm{T})$ \\
\hline 1 & 3.2298 \\
\hline 2 & 3.685 \\
\hline 3 & 3.8699 \\
\hline 4 & 3.9702 \\
\hline 5 & 4.0327 \\
\hline
\end{tabular}

Graph :3

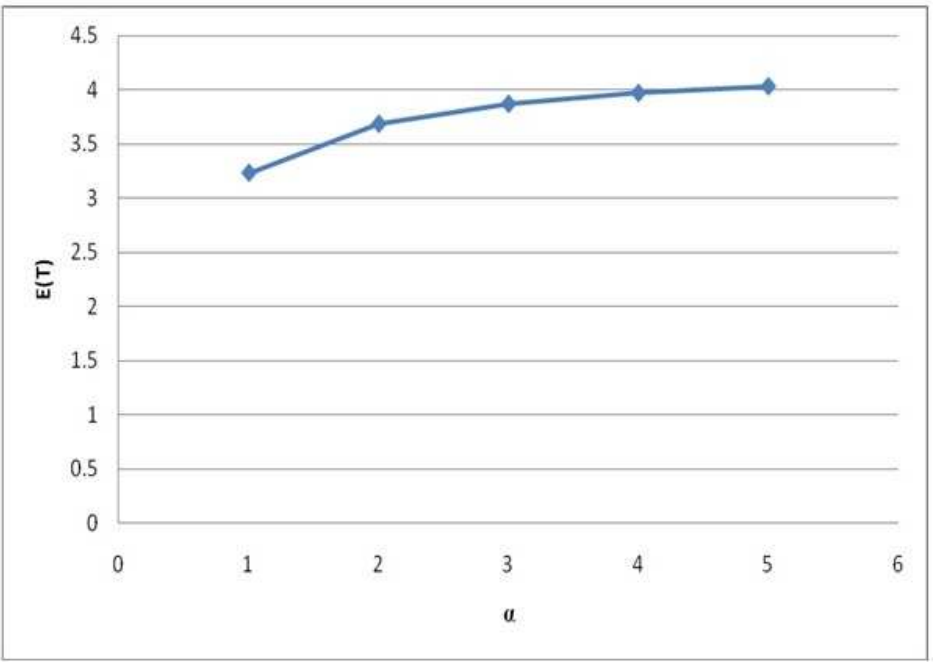


As a value of $\mu$ which is the parameter of the random variable representing the amount of depletion at every epoch of transfer of personnel increases then it is seen that $E(T)$ which is the expected time to recruitment increases. This is due to the fact that $Y$ is distributed as exponential with parameter $\mu$ and $E(Y)=\frac{1}{\mu}$

As $\mu$ increases $E(Y)$ namely the average amount of depletion decreases hence $E(T)$ namely time to recruitment increases.

As $R$, the correlation between the inter-decision time increases and hence the mean time to recruitment increases

\section{References}

[1] J.D. Esary, A.W. Marshall, F. Proschan, Shock models and wear processes, Auu. Probability, 1 (1973), 627-649.

[2] J. Gurland, Distribution of the maximum of the arithmetic mean of correlated random variables, Auu. Math. Statist., 26 (1955), 294-300.

[3] R. Sathiyamoorthi, R. Elangovan, Shock model approach to determine the expected time for recruitment, Journal of Decision and Mathematika Sciences, 3, No-s: 1-3 (1998).

[4] R. Sathiyamoorthi, S. Parthasarathy, On the expected time to recruitment in a two grade marketing organization, IAPQR Transactions, 27, No. 1 (2002).

[5] K. Usha, P. Leelathilagam, R. Ramanarayanan, A stochastic model for estimation of expected time to recruitment under two sources of depletion of man power, International Journal of Applied Mathematics, 23, No. 5 (2010). 
